\title{
Prévention de blessures et triathlon ${ }^{\text {is }}$
}

\section{Injury prevention and triathlon}

\author{
F. Delvaux \\ J.-L. Croisier \\ D. Sanfilippo \\ A. Gofflot \\ C. Tooth \\ J.-F. Kaux \\ B. Forthomme
}

Département des sciences de la motricité, université de Liège, CHU de Liège, Liège, Belgique

\section{RÉSUMÉ}

Bien que la littérature scientifique relative à la prévention de blessures dans le triathlon soit globalement pauvre, des lignes de conduite directement applicables sur le terrain peuvent être proposées. Les principales blessures du triathlète étant des lésions de surcharge des membres inférieurs (tendinopathies, fractures de fatigue, périostites), leurs facteurs de risque principaux sont un premier épisode lésionnel et une charge de travail inadaptée. Concrètement, les mesures préventives peuvent être regroupées selon différents axes : réaliser un bilan médical de pré-saison pour recenser tout élément susceptible de prédisposer à la survenue d'une blessure ultérieure ; monitorer et gérer la charge de travail ; prévoir une préparation physique incluant notamment un travail de core stability et de renforcement musculaire afin de préparer les organismes aux contraintes ; insister sur une qualité de gestuelles techniques dans chacune des trois disciplines; ne pas négliger d'éventuels autres facteurs de risque modifiables comme I'hygiène de vie ou la pratique d'échauffement de qualité. Cependant, il apparaît nécessaire de disposer de plus d'études sur le sujet pour pouvoir répondre encore plus efficacement aux besoins fondamentaux de la prévention lésionnelle dans le triathlon.

(c) 2019 Elsevier Masson SAS. Tous droits réservés.

\section{SUMMARY}

Despite a poor literature in the area of triathlon injury prevention, guidelines can be proposed for triathletes and their coaches. In triathlon, overuse injuries of lower limbs (tendinopathies, stress fractures, tibial stress syndromes) represent a large majority of all injuries. The most important risk factors are a previous history of injury and an inadequate workload. Actually, preventive measures should include: a pre-competition clinical assessment in order to identify every predisposing factor to a potential future injury; a monitoring and subsequent management of workload; physical preparation sessions with core stability and strength training; a high quality of technical movements in each of the three disciplines; a management of other potential risk factors such as inadequate life style or warm-up. Further studies are required in order to improve the quality of triathlon injury prevention.

(c) 2019 Elsevier Masson SAS. All rights reserved.

\section{INTRODUCTION}

Créé dans les années 1970, le triathlon a connu depuis lors un accroissement important de son nombre de participants. Ainsi, à l'heure actuelle, on estime à plus de 100000 le nombre de pratiquants qui réalisent au minimum une compétition de triathlon par année sur le territoire français. Ce sport exigeant, décliné selon de multiples distances sur chacune des trois disciplines, peut évidemment générer des blessures de l'appareil locomoteur. Dès lors, comme pour toute activité physique, une réflexion sur d'éventuelles actions préventives se justifie pleinement, notamment sur la base des dernières avancées importantes concernant l'évidence scientifique relative à la prévention de blessures. Pour offrir une efficacité

\section{MOTS CLÉS}

Prévention lésionnelle Triathlon

Surcharge

Facteurs de risque

\section{KEYWORDS}

Injury prevention Triathlon

Overuse

Risk factors 
optimale, la mise en place d'une stratégie de prévention de blessures débute classiquement par le choix d'un modèle théorique [1]. Le plus répandu d'entre eux est sans conteste la séquence de prévention, développé par van Mechelen et al. [2]. La première étape de ce modèle, qui en comporte quatre, consiste à recenser les données épidémiologiques telles que l'incidence et la sévérité des blessures au sein d'une population cible ; en deuxième lieu, il s'agit d'identifier et de comprendre les facteurs de risque et les mécanismes lésionnels des pathologies en question ; la troisième étape consiste en la mise en place effective des mesures préventives, et la quatrième étape mesure l'efficacité des mesures préventives introduites en répétant l'étape 1.

L'objectif de cet article est de réaliser une synthèse des actions préventives pouvant être appliquées aux triathlètes. Pour ce faire, nous avons adapté le modèle préventif théorique à la pratique du triathlon : après un passage en revue des blessures les plus fréquentes en triathlon ainsi que des facteurs de risque menant à celles-ci, une description des actions préventives concrètes et de leur efficacité sera développée.

\section{ÉPIDÉMIOLOGIE DES BLESSURES AU TRIATHLON}

Étonnamment, la littérature scientifique relative à l'épidémiologie des blessures du triathlète apparaît globalement très pauvre [3]. Tout comme pour l'ensemble des sports d'endurance, les pathologies de surcharge représenteraient environ $80 \%$ des blessures observées chez le/la triathlète [4-7]. Cependant, la majorité des études sur le sujet souffre de biais méthodologiques : design rétrospectif, durée courte de suivi, cohortes de faible importance, etc... Pour pallier à ces manques, Andersen et al. ont réalisé un suivi prospectif sur 174 triathlètes longue distance [8]. La prévalence de pathologies de surcharge durant les six mois de cette étude grimpait à $56 \%$; plus d'un tiers de ces athlètes (soit $20 \%$ de la population totale) a déclaré que leur blessure de surcharge a impliqué une réduction substantielle de leur volume habituel de travail, une baisse des performances ou encore une incapacité à participer à une compétition. Avec une prévalence de $25 \%$ de la totalité des lésions de surcharge, le genou est le site le plus souvent touché devant la jambe et la région lombaire (23\% chacune), l'épaule (20\%) et la cuisse (12\%). La durée des plaintes était en moyenne assez longue : celles-ci se prolongeaient sur 34 à $47 \%$ du temps de l'étude. Zwingenberger et al. [9] ont également réalisé un suivi prospectif d'une durée d'un an sur une cinquantaine de triathlètes et sont arrivés à la conclusion que la course à pied, le cyclisme et la natation seraient responsables respectivement de 50, 43 et $7 \%$ des blessures ; ces proportions apparaissent globalement équivalentes à celles retrouvées par Egermann et al. [10] quelques années plus tôt. Les localisations de blessures étaient réparties comme suit : $84 \%$ aux membres inférieurs (genou $33 \%$, jambe $24 \%$, pied $11 \%$, cheville $9 \%$, cuisse $7 \%$ ), $9 \%$ au tronc et $6 \%$ aux membres supérieurs (épaule, main). La nature des blessures de surcharge est rarement spécifiée dans les études prospectives [3]. Zwingenberger et al. [9] ont recensé que $22 \%$ des blessures étaient des abrasions/contusions, $46 \%$ des blessures tendineuses ou musculaires et $32 \%$ des lésions capsulaires et/ou ligamentaires. Parmi cette cohorte, on ne retrouve étrangement aucune fracture traumatique ou de stress. Neidel et al. [4], dans leur étude rétrospective sur des triathlètes allemands de haut niveau, identifient pourtant que les fractures de stress sont fréquentes; la totalité de ces fractures est située aux membres inférieurs, avec $52 \%$ aux métatarsiens, $19 \%$ au fémur, $10 \%$ au tibia, $10 \%$ à la fibula et $10 \%$ au calcanéum. En croisant ces données avec celles relatives aux blessures des coureurs à pied [11], nous pouvons estimer que les pathologies de surcharge des membres inférieurs sont essentiellement des tendinopathies achilléennes, patellaires ou glutéales, des syndromes fémoro-patellaires, des syndromes de friction de la bandelette ilio-tibiale, des fractures de stress, des périostites tibiales ou encore des aponévrosites plantaires.

Les lésions d'épaule s'avèrent principalement liées à la pratique de la natation, même si la fréquence lésionnelle des blessures d'épaule s'avère moindre par rapport aux autres sites anatomiques [12]. Une raison principale de ce faible taux lésionnel semble liée au temps moindre consacré à cette discipline lors des entraînements et des compétitions que celui dédié au vélo et à la course : il est seulement de $10 \%$ par rapport au temps total d'un Ironman [13].

Les lésions d'épaule apparaissent d'origine microtraumatique majoritairement, étant donné la répétition importante du cycle de bras en natation. Un triathlète de haut niveau exécute en moyenne 500000 cycles de bras en une saison d'entraînement. La biomécanique, lors de la pratique du crawl, reste par ailleurs primordiale dans la survenue de lésions [14], étant donné que tous les triathlètes utilisent ce style à l'entraînement et en compétition [13]. En effet, le crawl s'avère être le style le plus rapide et autorise l'athlète à ajuster sa trajectoire en levant la tête hors de l'eau pendant la course.

Au même titre que l'épaule du sportif d'armé-lancer, la natation en triathlon entraîne des tendinopathies de la coiffe des rotateurs (supra-épineux, subscapulaire et tendon du long biceps) liées au conflit intra-auriculaire et au conflit superficiel primaire ou secondaire [14]. Des lésions d'instabilité antéro-interne ou multidirectionnelle sont également décrites ainsi que des arthropathies acromio-claviculaires. La répétition de la gestuelle de type " overhead » lors de la pratique du crawl favorise ces lésions de surcharge.

Les lésions aiguës présenteraient une incidence de l'ordre de, respectivement, 0,97 à 1,02 blessures par 1000 heures de pratique à l'entraînement ou en compétition [8]. Environ 60 \% de ces lésions aiguës sont provoquées par des chutes à vélo et sont de gravité variable, allant de la simple contusion à des fractures multiples. Le reste des lésions aiguës (entorses de cheville, lésions musculaires de la cuisse ou de la jambe...) ne représentent que moins de $10 \%$ des blessures totales et surviennent quasi exclusivement durant la pratique de la course à pied.

\section{Facteurs de risque lésionnels des lésions de surcharge}

L'identification des facteurs de risque de lésions de surcharge liées à la pratique du triathlon ne peut se résumer à l'addition des facteurs de risque propres à chacune de ses trois composantes. Un(e) triathlète est, par exemple, moins à risque de développer une lésion de surcharge à l'épaule ou aux membres inférieurs qu'un nageur ou un coureur à pied de longue distance [15]. Dès lors, pour l'étude des facteurs de risque propres aux triathlètes, l'utilisation des données scientifiques relatives aux trois disciplines prises de manière isolée peut 
mener à des erreurs mais est, malheureusement, imposée partiellement par la pauvreté des études uniquement consacrées au triathlon.

\section{Facteurs de risque intrinsèques}

Les antécédents de blessures représentent le facteur de risque le plus important chez les triathlètes $[5,6,16]$. Plus précisément, en ce qui concerne les tendinopathies et les fractures de stress des membres inférieurs (indépendamment de la localisation), il est communément admis qu'un premier épisode accroît le risque de développer un épisode ultérieur $[17,18]$. Parmi les autres facteurs de risque intrinsèques de blessures de surcharge chez le triathlète, l'évidence scientifique peut être considérée comme faible quant à l'influence de l'âge, du genre, de mesures anthropométriques, de force musculaire, d'années d'expérience en triathlon ou encore de qualités biomécaniques sur la survenue des blessures [3]. Burns et al. [6] avaient identifié un taux de blessures supérieur chez les athlètes « supinateurs » à l'attaque du pied au sol en course à pied, mais ces résultats n'ont pas été confirmés par d'autres études. Le genre masculin ou féminin ne semble pas prédisposer à un degré d'exposition différent aux blessures, mais la nature des blessures pourrait partiellement dépendre du genre: les fractures de stress, tous sports confondus, apparaissent significativement plus fréquentes chez les femmes [19]. Ces fractures de stress sont généralement associées à la triade de l'athlète féminine, intégrée plus récemment dans le concept de relative energy deficiency in sport (RED-S) [20], qui se définit comme un déséquilibre entre les apports caloriques et la dépense énergétique, avec notamment des troubles menstruels et des effets potentiellement délétères sur l'os [19]. Bien que suggéré par certains auteurs [21], les tendinopathies des membres inférieurs, en particulier les tendinopathies achilléennes et patellaires ne semblent pas plus fréquentes chez les hommes que chez les femmes [22], mais ces données ne sont pas spécifiques aux triathlètes.

Même s'il existe une réelle spécificité de la pratique du triathlon, les facteurs intrinsèques des lésions d'épaule correspondent aux facteurs de risque intrinsèques identifiés chez les nageurs avec, néanmoins, une fréquence lésionnelle moindre [23]. Les facteurs de risque mis en évidence dans la littérature par les études prospectives concernent la modification de mobilité gléno-humérale, la dyskinésie scapulothoracique, les troubles posturaux ainsi que le manque de force développés par les rotateurs d'épaule. Walker et al. [23] observent qu'une modification de la rotation externe passive d'épaule mesurée à $90^{\circ}$ d'abduction frontale, sous la forme d'une augmentation $\left(>100^{\circ}\right.$ ) ou d'une diminution $\left(<93^{\circ}\right)$, majore le risque de douleur d'épaule. Les auteurs identifient une augmentation du risque de 8,1 et $12,5 \%$ chez les nageurs présentant cette modification (perte ou gain) de la mobilité en rotation externe. Bien que l'hyperlaxité soit fréquemment décrite chez le nageur, il n'existe pas, à notre connaissance, d'études prospectives liant cette majoration de la laxité gléno-humérale à la survenue lésionnelle.

McKenna et al. [24], lors d'un suivi longitudinal de 46 nageurs, ont mis en évidence la présence d'une dyskinésie scapulaire au repos (écartement majoré de la scapula par rapport à la colonne) et d'une antéprojection des épaules en début de saison comme facteurs de risque de scapulalgies. Tate et al. [25] associent la faiblesse des rotateurs internes et du trapèze moyen, la raideur d'épaule en flexion et la raideur du petit pectoral à la douleur d'épaule chez 235 nageuses de compétition. Swanik et al. [26] observent une diminution de l'incidence des scapulalgies après un renforcement des rotateurs interne et externe de la coiffe. Actuellement, peu d'évidence scientifique existe sur l'influence réelle d'un déséquilibre de force au sein de la coiffe des rotateurs sur la lésion de l'épaule sportive.

Les triathlètes présentant une posture en antéprojection des épaules et un manque de mobilité en extension du thorax semblent plus à risque de lésions de type tendinopathies de la coiffe, étant donné, en particulier, la position de l'épaule lors du retour aérien en crawl [14].

\section{Facteurs de risque extrinsèques}

La charge de travail représente probablement le facteur de risque extrinsèque de blessure le plus puissant. Forte d'un intérêt scientifique important sur le sujet durant ces dernières années, une récente revue systématique a clairement identifié une relation entre charge de travail et survenue de blessures, indépendamment du type de lésion ou de sport [27]. À notre connaissance, une seule étude prospective a tenté d'établir un éventuel lien entre charge de travail et survenue de blessures spécifiquement chez le triathlète $(n=155)$ [5]. Aucune association entre blessure et charge (évaluée via : distance hebdomadaire de natation, cyclisme et course à pied ; nombre de sessions ou d'heures hebdomadaires ; intensité des sessions) n'a cependant été mise en évidence. De nouvelles études spécifiques aux triathlètes seraient souhaitables, en intégrant notamment des paramètres de charge de travail tels le session/s rating of perceived exertion (SRPE), le ratio charge aiguë/charge chronique (CACR) ou d'autres mesures de charge interne ou externe. Sans pouvoir valider solidement cette hypothèse d'un point de vue scientifique, les blessures de surcharge du triathlète comme les tendinopathies des membres inférieurs ou les fractures de stress sont très probablement liées à une inadéquation entre la charge d'entraînement et la capacité de ces tissus à tolérer celle-ci.

À côté de la charge de travail, il est très difficile d'identifier d'autres paramètres extrinsèques qui seraient associés étroitement avec la survenue de blessures en triathlon. La pratique du triathlon longue distance/Ironman, qui engendre de facto une charge de travail plus élevée, serait plus génératrice de blessures de surcharge que la pratique de distances inférieures [10]. Les triathlètes présentant des lésions aiguës d'épaule ont, par ailleurs, consacré un temps significativement plus important à l'entraînement avec charges ; ceux décrivant davantage de lésions chroniques ont participé à plus de courses que les athlètes sains [28].

Sans évidence scientifique probante, certains autres facteurs sont régulièrement cités [3] comme l'absence d'échauffement, de cool down, de stretching ou encore de coach ; la qualité de la surface de course ; une alimentation ou une hydratation inadéquate, le port de chaussures de course inadéquates.

\section{QUELLES MESURES CONCRÈTES SUR LE TERRAIN ?}

À notre connaissance, aucune étude de haute qualité n'a évalué l'influence d'une stratégie préventive sur la survenue de blessures dans le triathlon. Pour rester dans la lignée du modèle de van Mechelen et al. [2], une approche cohérente 
serait de proposer des mesures préventives sur la base de facteurs de risque lésionnels, en priorité les facteurs de risque démontrés scientifiquement. Cependant, comme mentionné précédemment, l'évidence scientifique est globalement pauvre sur le sujet et les recommandations de bonnes pratiques préventives doivent donc s'inspirer également des stratégies qui ont prouvé leur efficacité dans chacune des trois disciplines du triathlon ainsi que dans d'autres sports. Enfin, l'expérience de terrain avec des triathlètes pourra compléter ces recommandations. Nous pouvons, dès lors, considérer qu'une stratégie de prévention de blessures devrait comprendre les éléments suivants:

\section{Un bilan médical de pré-saison}

Le bilan médical de pré-saison a pour objectif de recenser tout élément susceptible de prédisposer à la survenue d'une blessure ultérieure. La prise en compte des antécédents lésionnels constitue une étape indispensable de ce bilan, au regard de l'influence importante d'un premier épisode lésionnel sur la survenue d'une récidive [5]. Spécifiquement chez la triathlète féminine, l'identification d'un éventuel RED-S apparaît primordiale. Des tests de provocation comme une évaluation de la laxité de cheville ou d'épaule pourraient conduire, le cas échéant, à proposer un programme pertinent de remédiation ou de rééducation, d'un point de vue préventif. L'identification d'éventuels troubles de la statique, des restrictions de la mobilité articulaire ou de l'extensibilité musculaire ainsi que l'observation de la dyskinésie scapulothoracique devraient aussi faire partie de cet examen de base et justifier une remédiation. Des tests cliniques spécifiques à chaque articulation, même si certains souffrent d'un manque de spécificité et de sensibilité, ou encore l'imagerie, permettent de guider le diagnostic en cas d'éventuelle lésion pré-existante [29].

Ce bilan peut être avantageusement complété par l'utilisation d'outils de pointe comme le dynamomètre isocinétique ou encore d'autres évaluations de terrain ou de laboratoire.

\section{Une quantification et une gestion de la charge de travail}

Quantifier la charge de travail et la moduler en fonction des échéances compétitives est un mécanisme utilisé depuis toujours par les entraîneurs pour amener un athlète en forme optimale le jour J. C'est également une composante centrale de toute stratégie préventive, quelle que soit la discipline sportive [30]. L'objectif consiste donc à trouver un équilibre entre la charge exercée sur l'athlète et la capacité de ses tissus à tolérer cette charge. Selon Mujika [31], il n'existe pas de méthode " gold standard " pour évaluer cette charge de travail dans les sports d'endurance. Le choix d'utiliser une méthode en particulier dépend, en premier lieu, des qualités métrologiques de cette méthode, mais aussi de facteurs comme la faisabilité pratique/l'accessibilité ou le rapport coût/efficacité. Classiquement, on distingue la charge externe de la charge interne : si la charge externe peut se définir comme tout stimulus appliqué à un athlète, indépendamment de ses caractéristiques internes [32], la charge interne correspond à la réponse d'un individu à la charge externe imposée [33]. Typiquement pour la pratique du triathlon, la comptabilisation de la durée hebdomadaire totale passée en piscine, à vélo ainsi qu'en course à pied et dans tout autre entraînement (musculation...) représente une première étape indispensable de quantification de la charge externe. Celle-ci serait idéalement complétée d'une mesure précisant les objectifs de chaque session ainsi que des distances parcourues. L'évaluation de la charge interne, indispensable au même titre que la charge externe, se calcule le plus fréquemment au moyen du SRPE qui équivaut au produit de la durée d'effort (en minutes) par l'intensité ressentie par l'athlète (sur une échelle de 0 à 10) pour chaque session sportive. Un nombre d'unités arbitraires (UA) est ainsi obtenu et peut être comptabilisé semaine après semaine. D'autres mesures de la charge interne peuvent également s'avérer utiles : un suivi de fréquence cardiaque (FC); un ratio FC/RPE ; le training impulse (TRIMP) et ses dérivés ; etc... Autre facette de la charge interne, la charge " émotionnelle et perceptive ", concernant les périodes hors entraînements ou compétitions, peut aussi être instructive. Celle-ci peut être approchée via certains questionnaires de bien-être, de stress ou de qualité de récupération comme le Recovery Stress Questionnaire for Athletes (REST-Q) ou le Profile of Mood States (POMS).

La grande difficulté réside principalement dans l'interprétation de ces données : quelles valeurs-seuils à ne pas dépasser? Quelle progressivité dans la charge semaine après semaine? À partir de quelles valeurs doit-on prévoir des phases de récupération ?... La littérature spécialisée ne nous renseignant que trop peu sur ces sujets pourtant essentiels, nous pouvons nous fier sur notre expérience pratique ainsi que sur les avis d'experts du domaine. Ainsi, Drew et Finch suggèrent de suivre sept grands principes pour gérer efficacement la charge de travail dans un but préventif [30] :

- établir une charge de travail moyenne sur une durée préchoisie en fonction de la planification (mensuelle, cycles de 3 à 12 semaines...) qui servira de ligne de base pour comparaison ultérieure ;

- réduire les variations importantes de charge entre deux semaines consécutives ;

- définir une charge hebdomadaire et mensuelle « plafond » et ne pas la dépasser;

- assurer une charge de travail minimale pour éviter une baisse de la capacité des tissus à tolérer cette charge ;

- répartir correctement la charge durant une même semaine ;

- s'assurer que la charge de travail est proportionnelle aux demandes de la discipline ;

- monitorer la charge durant la période précédant une blessure afin de pouvoir agir directement sur cette charge dès la déclaration des symptômes.

\section{Une préparation physique}

La préparation physique du triathlète comprend plusieurs éléments dont l'objectif commun est d'entraîner le corps aux efforts spécifiques en triathlon. Un travail de core stability apparaît indispensable, notamment au regard de l'étude récente de De Blaiser et al. [34] qui a identifié qu'une faiblesse du contrôle postural dynamique ainsi que de la force et de l'endurance des muscles du tronc (paramètres qui définissent cette notion de core stability) était associée à un risque accru de développer des lésions de surcharge aux membres inférieurs parmi une population athlétique. Un renforcement global des membres supérieurs et inférieurs permet d'améliorer la capacité musculo-tendineuse à résister à la charge imposée. Aux membres inférieurs, on sait désormais qu'un entraînement de la force revêt un caractère préventif de lésions tant aiguës que de surcharge [35]. À côté des exercices classiques 
de renforcement des muscles fléchisseurs et extenseurs des trois grandes articulations du membre inférieur, un renforcement spécifique des muscles de la hanche, en particulier des abducteurs de hanche, ne doit pas être négligé : il est probable qu'une faiblesse de ces abducteurs de hanche favorise la survenue d'un syndrome de friction de la bandelette ilio-tibiale [36] ou d'un syndrome fémoro-patellaire [37].

De plus, à partir de la description des facteurs de risque de lésions de l'épaule liées à la pratique du triathlon et à l'entraînement en natation, la correction posturale par l'étirement du petit pectoral notamment, l'amélioration du contrôle moteur et de l'équilibre de force des interscapulaires et des rotateurs d'épaule, la normalisation de la mobilité et le contrôle de l'instabilité gléno-humérale restent les objectifs principaux à prendre en compte dans la gestion des épaules du triathlète, sur la base du bilan spécifique de début de saison ou lors d'une symptomatologie particulière [38].

\section{Une gestuelle de qualité}

Idéalement supervisées par un entraîneur, les sessions d'entraînement devraient être réalisées selon une gestuelle technique propre. En effet, dans de nombreuses disciplines sportives, une pauvreté des qualités gestuelles a été associée à un stress mécanique supérieur, voire - étape supplémentaire - à un risque accru de blessures. Ainsi, en course à pied, l'attaque du sol par l'avant-pied, comparativement à une attaque talon, est associée à un stress mécanique fémoropatellaire inférieur [39], tout comme une augmentation de la fréquence de pas qui, de facto, engendre une réduction de l'amplitude de foulée. En natation, la répétition de gestes " overhead " lors du mouvement de nage libre, avec la particularité de nécessiter une extension de la tête lors de la nage en eaux libres chez le triathlète, provoque des microtraumatismes d'épaule qui peuvent être majorés si la gestuelle est impropre [13]. Enfin, le positionnement sur vélo doit être judicieusement adapté à l'athlète, ce qui, outre le fait d'améliorer les qualités aérodynamiques et le rendement, pourraient contribuer à réduire la survenue de lésions de surcharge, notamment au niveau du dos [40].

\section{Autres éléments}

Plusieurs facteurs, non spécifiques au triathlète, ne peuvent être négligés dans l'optique d'une prévention optimale : une hygiène de vie correcte (incluant notamment sommeil, hydratation et alimentation satisfaisants) [41], la pratique d'un échauffement de qualité [42] ou encore le port de bonnes chaussures [43]. Si la pratique régulière d'étirements ne peut être considérée comme un élément préventif de première importance [43]. Néanmoins, nous recommandons aux athlètes présentant des restrictions de mobilité aux membres inférieurs de s'étirer régulièrement afin de retrouver une souplesse satisfaisante.

\section{CONCLUSION}

Les recommandations à fournir aux triathlètes et à leurs entraîneurs en termes de prévention de blessures ne peuvent, à l'heure actuelle, se baser sur une évidence scientifique forte. Néanmoins, en appliquant une démarche théorique basée sur un modèle préventif de qualité, nous pouvons proposer des lignes de conduite directement applicables sur le terrain. Les principales blessures du triathlète étant des lésions de surcharge des membres inférieurs (tendinopathies, fractures de fatigue, périostites), leurs facteurs de risque principaux sont un premier épisode lésionnel et une charge de travail inadaptée. Concrètement, les mesures préventives peuvent être regroupées selon différents axes : réaliser un bilan médical de présaison pour recenser tout élément susceptible de prédisposer à la survenue d'une blessure ultérieure ; monitorer et gérer la charge de travail ; prévoir une préparation physique, notamment en incluant un travail de core stability et de renforcement musculaire afin de préparer les organismes à la charge de travail imposée ; insister sur une qualité de gestuelle technique dans chacune des trois disciplines; ne pas négliger d'éventuels autres facteurs de risque modifiables comme l'hygiène de vie ou la pratique d'un échauffement de qualité. Cependant, il apparaît nécessaire de disposer de plus d'études sur le sujet pour pouvoir répondre encore plus efficacement aux besoins fondamentaux de la prévention lésionnelle dans le triathlon.

Déclaration de liens d'intérêts

Les auteurs déclarent ne pas avoir de liens d'intérêts.

\section{RÉFÉRENCES}

[1] Delvaux F, Kaux J-F, Forthomme B, Croisier J-L. La prévention des blessures sportives : modèles théoriques et éléments-clés d'une stratégie efficace. J Traumatol Sport 2018;35(3):152-7.

[2] van Mechelen W, Hlobil H, Kemper HC. Incidence, severity, aetiology and prevention of sports injuries. A review of concepts. Sports Med 1992;14(2):82-99

[3] Gosling CM, Gabbe BJ, Forbes AB. Triathlon-related musculoskeletal injuries: the status of injury prevention knowledge. J Sci Med Sport 2008;11(4):396-406.

[4] Neidel P, Wolfram P, Hotfiel T, Engelhardt M, Koch R, Lee G, et al. Cross-sectional investigation of stress fractures in German elite triathletes. Sports (Basel) 2019;7:1-10.

[5] Korkia PK, Tunstall-Pedoe DS, Maffulli N. An epidemiological investigation of training and injury patterns in British triathletes. $\mathrm{Br}$ J Sports Med 1994;28(3):191-6.

[6] Burns J, Keenan A-M, Redmond AC. Factors associated with triathlon-related overuse injuries. J Orthop Sports Phys Ther 2003;33(4):177-84.

[7] Vleck VE, Bentley DJ, Millet GP, Cochrane T. Triathlon event distance specialization: training and injury effects. J Strength Cond Res 2010;24(1):30-6.

[8] Andersen CA, Clarsen B, Johansen TV, Engebretsen L. High prevalence of overuse injury among iron-distance triathletes. $\mathrm{Br} \mathrm{J}$ Sports Med 2013;47(13):857-61.

[9] Zwingenberger S, Valladares RD, Walther A, Beck H, Stiehler $M$, Kirschner S, et al. An epidemiological investigation of training and injury patterns in triathletes. J Sports Sci 2014;32(6):583-90.

[10] Egermann M, Brocai D, Lill CA, Schmitt H. Analysis of injuries in long-distance triathletes. Int J Sports Med 2003;24(4):271-6.

[11] Lopes AD, Hespanhol Junior LC, Yeung SS, Costa LOP. What are the main running-related musculoskeletal injuries? A systematic review. Sports Med 2012;42(10):891-905.

[12] Galera O, Gleizes-Cervera S, Pillard F, Rivière D. Prevalence of injuries in triathletes from a French league. Apunts Med Esport 2012;47(173):9-15. 
[13] Bales J, Bales K. Swimming overuse injuries associated with triathlon training. Sports Med Arthrosc Rev 2012;20(4):196-9.

[14] McHardy A, Pollard H, Fernandez M. Triathlon injuries: a review of the literature and discussion of potential injury mechanisms. Clin Chiropr 2006;9(3):129-38.

[15] Migliorini S. Risk factors and injury mechanism in Triathlon. J Hum Sport Exercise 2011;6(2):1-6.

[16] Villavicencio AT, Burneikiene S, Hernandez TD, Thramann J. Back and neck pain in triathletes. Neurosurg Focus 2006;21(4):E7.

[17] O'Neill S, Watson PJ, Barry S. A delphi study of risk factors for achilles tendinopathy - opinions of world tendon experts. Int $J$ Sports Phys Ther 2016;11(5):684-97.

[18] Duarte Sosa D, Fink Eriksen E. Women with previous stress fractures show reduced bone material strength. Acta Orthop 2016;87(6):626-31.

[19] Rizzone KH, Ackerman KE, Roos KG, Dompier TP, Kerr ZY. The epidemiology of stress fractures in collegiate student-athletes, 2004-2005 through 2013-2014 academic years. J Athl Train 2017;52(10):966-75.

[20] Statuta SM, Asif IM, Drezner JA. Relative energy deficiency in sport (RED-S). Br J Sports Med 2017;51(21):1570-1.

[21] Taunton JE, Ryan MB, Clement DB, McKenzie DC, Lloyd-Smith DR, Zumbo BD. A retrospective case-control analysis of 2002 running injuries. Br J Sports Med 2002;36(2):95-101.

[22] Albers IS, Zwerver J, Diercks RL, Dekker JH, Van den AkkerScheek I. Incidence and prevalence of lower extremity tendinopathy in a Dutch general practice population: a cross-sectional study. BMC Musculoskelet Disord 2016;17(16):1-6.

[23] Walker H, Gabbe B, Wajswelner H, Blanch P, Bennell K. Shoulder pain in swimmers: a 12-month prospective cohort study of incidence and risk factors. Phys Ther Sport 2012;13(4):243-9.

[24] McKenna L, Straker L, Smith A. Can scapular and humeral head position predict shoulder pain in adolescent swimmers and nonswimmers? J Sports Sci 2012;30(16):1767-76.

[25] Tate A, Turner GN, Knab SE, Jorgensen C, Strittmatter A, Michener LA. Risk factors associated with shoulder pain and disability across the lifespan of competitive swimmers. J Athl Train 2012;47(2):149-58.

[26] Swanik KA, Lephart SM, Swanik CB, Lephart SP, Stone DA, Fu $\mathrm{FH}$. The effects of shoulder plyometric training on proprioception and selected muscle performance characteristics. J Shoulder Elbow Surg 2002;11(6):579-86.

[27] Eckard TG, Padua DA, Hearn DW, Pexa BS, Frank BS. The relationship between training load and injury in athletes: a systematic review. Sports Med 2018;48(8):1929-61.

[28] Schorn D, Vogler T, Gosheger G, Schneider K, Klingebiel S, Rickert C, et al. Risk factors for acute injuries and overuse syndromes of the shoulder in amateur triathletes - A retrospective analysis. PLoS One 2018;13(6):e0198168.
[29] Hill L, Collins M, Posthumus M. Risk factors for shoulder pain and injury in swimmers: a critical systematic review. Phys Sports Med 2015;43(4):412-20.

[30] Drew MK, Finch CF. The relationship between training load and injury, illness and soreness: a systematic and literature review. Sports Med 2016;46(6):861-83.

[31] Mujika I. Quantification of training and competition loads in endurance sports: methods and applications. Int J Sports Physiol Perform 2017;12(Suppl. 2):S29-17.

[32] Wallace LK, Slattery KM, Impellizzeri FM, Coutts AJ. Establishing the criterion validity and reliability of common methods for quantifying training load. J Strength Cond Res 2014;28(8):2330-7.

[33] Halson SL. Monitoring training load to understand fatigue in athletes. Sports Med 2014;44(Suppl. 2):S139-47.

[34] De Blaiser C, De Ridder R, Willems T, Vanden Bossche L, Danneels L, Roosen P. Impaired core stability as a risk factor for the development of lower extremity overuse injuries: a prospective cohort study. Am J Sports Med 2019;47(7):1713-21.

[35] Lauersen JB, Andersen TE, Andersen LB. Strength training as superior, dose-dependent and safe prevention of acute and overuse sports injuries: a systematic review, qualitative analysis and meta-analysis. Br J Sports Med 2018;52(24):1557-63.

[36] Mucha MD, Caldwell W, Schlueter EL, Walters C, Hassen A. Hip abductor strength and lower extremity running-related injury in distance runners: a systematic review. J Sci Med Sport 2017;20 (4):349-55.

[37] Ramskov D, Barton C, Nielsen RO, Rasmussen S. High eccentric hip abduction strength reduces the risk of developing patellofemoral pain among novice runners initiating a self-structured running program: a 1-year observational study. J Orthop Sports Phys Ther 2015;45(3):153-61.

[38] Struyf F, Tate A, Kuppens K, Feijen S, Michener LA. Musculoskeletal dysfunctions associated with swimmers' shoulder. $\mathrm{Br} \mathrm{J}$ Sports Med 2017;51(10):775-80.

[39] Dos Santos AF, Nakagawa TH, Serrao FV, Ferber R. Patellofemoral joint stress measured across three different running techniques. Gait Posture 2019;68:37-43.

[40] Mellion MB. Common cycling injuries. Management and prevention. Sports Med 1991;11(1):52-70.

[41] von Rosen P, Frohm A, Kottorp A, Friden C, Heijne A. Too little sleep and an unhealthy diet could increase the risk of sustaining a new injury in adolescent elite athletes. Scand J Med Sci Sports 2017;27(11):1364-71.

[42] Vriend I, Gouttebarge V, Finch CF, van Mechelen W, Verhagen EALM. Intervention strategies used in sport injury prevention studies: a systematic review identifying studies applying the haddon matrix. Sports Med 2017;47(10):2027-43.

[43] Yeung SS, Yeung EW, Gillespie LD. Interventions for preventing lower limb soft-tissue running injuries. Cochrane Database Syst Rev 2011;6(7):CD001256. 